\title{
Energy restriction: stepping stones towards cancer therapy
}
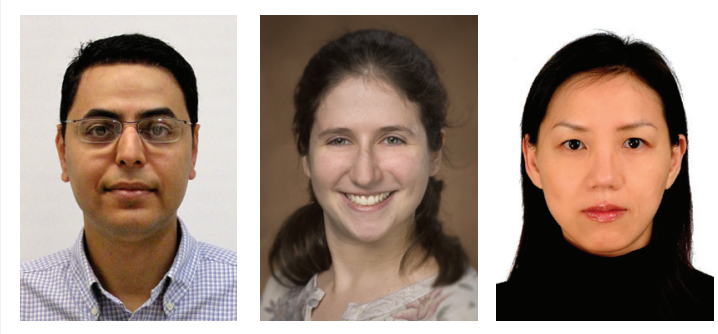

“...transformed cells exhibit

a high demand for

glucose with very limited

flexibility for modifying

their means of ATP

generation..."

\section{Hany A Omar ${ }^{1,2}$, Lisa Berman-Booty ${ }^{3}$ \& Jing-Ru Weng*4}

'Department of Pharmacology, Faculty of Pharmacy, Beni-Suef University, 62514, Egypt

${ }^{2}$ College of Pharmacy, Taif University, Taif, KSA

${ }^{3}$ Division of Medicinal Chemistry, College of Pharmacy \& Comprehensive Cancer Center, The Ohio State

University, Columbus, OH 43210, USA

4Department of Biological Science \& Technology, China Medical University, Taichung, 40402, Taiwan

*Author for correspondence: Tel.: +886 422053366 ext. 2517 = Fax: +886 422071507 = columnster@gmail.com

The growing interest in utilizing energy restriction without malnutrition as an approach for cancer therapy and prevention is due to the promising results from animal and human trials. The principle of targeting energy metabolism as an antitumor strategy relies on the differences in the ways that normal and transformed cells generate energy. Unlike non-neoplastic cells, transformed cells exhibit a high demand for glucose with very limited flexibility for modifying their means of ATP generation in response to changing environmental conditions and energy source availability. Therefore, cancer cells are exquisitely vulnerable to energy restriction. However, it is extremely difficult to implement chronic energy restriction through actual dietary caloric restriction as an antitumor strategy in humans.

\section{Targeting cancer metabolism by energy restriction}

In the November 2010 issue of Future Oncology, we reviewed the development of small molecule agents that function as energy-restriction mimetic agents (ERMAs) and target various aspects of energy metabolism [1]. Here, we provide updates regarding energy-restriction research to date and a number of the ERMAs discussed in our previous editorial. Additionally, we discuss metabolic enzymes and signaling pathways that have recently been reported to be modulated by energy restriction.

Research has shown that energy restriction suppresses the clonal expansion of transformed cells by limiting survival factors required for the regulation of cell proliferation, cell cycle control and angiogenesis [2]. Many newly developed
ERMAs induce cellular responses that mimic actual dietary energy restriction.

\section{AMPK activators: an update}

The induction of cell cycle senescence by agents that activate AMPK has been studied extensively in the context of tumor cells growth inhibition. By activating AMPK, these agents mimic a low intracellular energy state and inhibit the proliferation of cancer cells in vitro. AMPK activation suppresses cancer cell growth either by inhibiting mTOR-dependent translation initiation [3] or by phosphorylating and inactivating acetylCoA carboxylase (ACC). ACC inactivation is accompanied by the suppression of fatty acid synthase, which leads to growth inhibition by blocking lipogenesis [4]. In vivo, treatment of diabetic patients with metformin, a widely used antidiabetic agent and an AMPK activator, reduces cancer-related mortality in this patient population [5]. This effect of metformin is independent of blood glucose levels, since other antidiabetic agents failed to show an anticancer effect [6]. At the molecular level, metformin inhibits mitochondrial complex I in the liver, thereby interfering with ATP production and results in a low ATP state and energy depletion that activates AMPK [7]. Recent studies have explored metformin's ability to target trastuzumab-resistant breast cancer cells via inhibition of erbB2/IGF-1 receptor interactions [8]. Additionally, an epidemiological study of the long-term use of metformin found that such use was associated with a decreased risk of ovarian cancer [9].

Aminoimidazole carboxamide ribonucleotide (AICAR), another widely used AMPK activator,

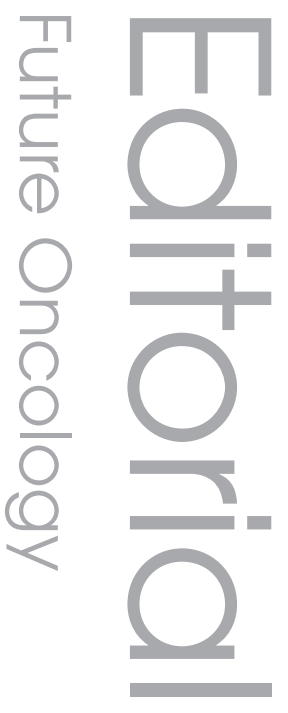

\section{Keywords}

- AMPK $=$ cancer therapy

- energy restriction-mimetic

agents $=$ hexokinase $\|$ m tumor

metabolism = Warburg effect

Future $\because$ Medicine part of 
has been extensively studied in many cancer types [10]. In addition to activating AMPK, AICAR increases $\mathrm{S}$-phase cell-cycle arrest and apoptosis, thereby suppressing cell proliferation [10].

\section{Resveratrol: modulation of the cellular 'energy-sensing' machinery by an ERMA} Resveratrol was one of the earliest identified ERMAs. It modulates tumor cell proliferation and protein translation via Sirtuin type 1 (SIRT1)-dependent activation of AMPK [11]. Resveratrol activates AMPK secondary to inducing SIRT1 expression via elevation of the cellular $\mathrm{NAD}^{+} / \mathrm{NADH}$ ratio. Studies utilizing melanoma cells found that AMPK-mediated growth inhibition was achieved via inactivation of the RAF-MEK-ERK mitogen-activated protein kinase pathway [10].

\section{Thiazolidinedione-derived ERMAs as a 'multi-pronged' approach to target tumor energy metabolism}

Research has shown that the thiazolidinedione (TZD) peroxisome proliferator-activated receptor (PPAR) agonists, troglitazone and ciglitazone, are able to mimic glucose starvation and elicit cellular responses characteristic of energy restriction independent of the activation of PPAR $\gamma$ [12]. Starvation-associated responses induced by the TZD derivatives include reduction of the glycolytic rates and intracellular levels of NADH and lactate, transient induction of the silent information regulator 1 gene, activation of AMPK and endoplasmic reticulum stress. The interplay among these responses likely culminates in autophagy and apoptosis. Ciglitazone was used as a scaffold to develop a novel ERMA, OSU-CG12 (CG-12), which has shown increased antiproliferative potency. In addition to inducing the starvation responses listed above, CG-12 is able to induce apoptosis in cancer cells through epigenetic activation of Krueppel-like factor 6 (KLF6) tumor suppressor gene expression [13].

When OSU-CG5 (CG-5), a newly developed TZD-derived ERMA, is combined with glucose deprivation, the expression levels of a number of DNA methylation-silenced tumor suppressor genes are upregulated, and the expression levels of tumor/invasion-promoting genes are downregulated. Upregulated genes included GADD45a, GADD45b, IGFBP3, LAMB3, $B A S P 1, G P X 3$ and GSTP1, and downregulated genes included CD44, S100A4 and TACSTD2 [14]. The epigenetic effects of CG-5 are mediated through transcriptional repression of DNA methyltransferase 1 , associated with reduced expression of Sp1 and E2F1. These data reveal the epigenetic effect of ERMAs on DNA methylation-silenced tumor suppressor genes.

66...energy restriction suppresses the clonal expansion of transformed cells by limiting survival factors required for the regulation of cell proliferation, cell cycle control and angiogenesis."

By better mimicking actual glucose starvation, it would seem that an ERMA, such as CG-12 or CG-5, that is able to induce multiple components of the starvation-associated response would offer advantages over an ERMA that targets only one component of this response. Initial studies show this to be the case in vitro, with CG-12 exhibiting higher antiproliferative activity than resveratrol in the human prostate cancer LNCaP and the human breast cancer MCF-7 cell lines [12] However, further studies are needed to compare their in vivo tumor growth suppressive activities and better delineate the full mechanism of action of CG-12 and CG-5. Based on the research thus far, these novel ERMAs and their derivatives offer tremendous potential as chemopreventive and/or chemotherapeutic agents.

\section{Hexokinase II inhibitors: an update}

Hexokinase II (HK II), the first enzyme in glycolysis, is able to bolster the energy supply of malignant cells and protect cancer cells against apoptosis through direct interaction with mitochondrial voltage dependent anion channel 1 (VDAC1) [15]. Inhibitors of mitochondrial-bound HK II such as lonidamine, sensitize cancer cells to apoptosis by facilitating the docking of the pro-apoptotic protein Bax on VDAC1 [16]. Furthermore, lonidamine is reported to inhibit colon fibrosarcoma cell migration and invasion [17] and it counteracts multidrug resistance in human breast and ovarian tumor cells when combined with paclitaxel [18]. Clinical trials have shown that the combination of lonidamine with other chemotherapeutic agents is active against advanced non-small-cell lung cancer and ovarian cancer [19]. The pyruvate analog 3-bromopyruvate is another HK II inhibitor and an alkylating agent. It has demonstrated suppressive activities against hepatocellular carcinoma [20], colon cancer [21] and breast cancer [22], both in vitro and in vivo.

\section{Opportunities \& challenges}

The use of ERMAs as a cancer therapy is limited by our incomplete knowledge of the 
regulation of metabolic pathways that facilitate cell proliferation [23]. Additionally, cancer cells exhibit heterogeneous metabolic alterations as a result of the tumor microenvironment, which affects tumor vasculature and results in gradients of nutrients, oxygen and $\mathrm{pH}$ [24]. Despite these limitations, agents that target tumor cell metabolism have been used successfully in human cancer therapy. For example, antimetabolites such as nucleoside analogues, hydroxyurea and antifolate drugs were among the earliest agents that targeted tumor metabolism and have been used extensively in the treatment of diverse human cancers. Additionally, L-asparaginase and analogous agents have been successfully used to treat acute lymphoblastic leukemia and related lymphomas. These agents were designed to specifically reduce levels of glutamine, a crucial nutrient for acute lymphoblastic leukemia and many cancer cells [25].

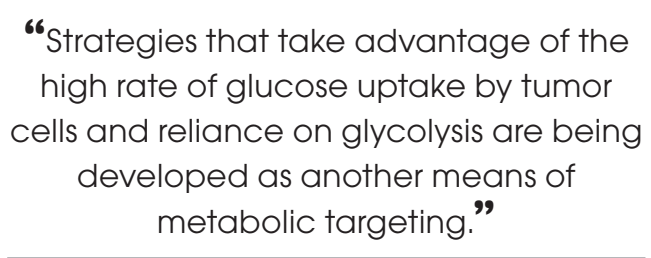

Strategies that take advantage of the high rate of glucose uptake by tumor cells and reliance on glycolysis are being developed as another means of metabolic targeting. For example, $\beta$-D-glucosylisophosphoramide mustard is an alkylating agent in which isophosphoramide mustard is covalently linked to $\beta$-D-glucose. Linking the isophosphoramide mustard to $\beta$-D-glucose enables glufosfamide to be selectively targeted to tumor sites [26].

The major challenge in the development of anticancer drugs that target cell metabolism is the potential toxicity to normal cells. However, the redundant metabolic pathways in normal cells and over-reliance of cancer cells on specific metabolic pathways can provide a safety threshold [24]. Genetic changes that result in the stringent reliance of cancer cells on few specific metabolic pathways (like aerobic glycolysis) could potentially be used as targets for future chemotherapeutic drug development. Although the use of ERMAs as chemotherapeutic agents is still in its infancy, as the intricacies of cancer energy metabolism are further elucidated, we foresee this field flourishing and becoming a mainstay in the fight against cancer.

\section{Financial \& competing interests disclosure \\ This work was supported by grants from the Taiwan Department of Health, China Medical University Hospital Cancer Research of Excellence (DOH1O1- TD-C-111-005) and the National Science Council of the Republic of China (NSC 101-2320-B-039- 029-MY2). The authors have no other relevant affili- ations or financial involvement with any organization or entity with a financial interest in or financial conflict with the subject matter or materials discussed in the manuscript apart from those disclosed. \\ No writing assistance was utilized in the production of this manuscript.}

\section{References}

1. Omar HA, Berman-Booty L, Kulp SK, Chen CS. Energy restriction as an antitumor target. Future Oncol. 6(11), 1675-1679 (2010).

2. Thompson HJ, Zhu Z, Jiang W. Dietary energy restriction in breast cancer prevention. J. Mammary Gland Biol. Neoplasia 8(1), 133-142 (2003).

3. Dowling RJ, Zakikhani M, Fantus IG, Pollak M, Sonenberg N. Metformin inhibits mammalian target of rapamycin-dependent translation initiation in breast cancer cells. Cancer Res. 67(22), 10804-10812 (2007).

4. Guo D, Hildebrandt IJ, Prins RM et al. The AMPK agonist AICAR inhibits the growth of EGFRvIII-expressing glioblastomas by inhibiting lipogenesis. Proc. Natl Acad. Sci. USA 106(31), 12932-12937 (2009).

5. Berstein LM, Boyarkina MP, Teslenko SY. Familial diabetes is associated with reduced risk of cancer in diabetic patients: a possible role for metformin. Med. Oncol. 29(2), 1308-1313 (2012).

6. Bowker SL, Majumdar SR, Veugelers P, Johnson JA. Increased cancer-related mortality for patients with type 2 diabetes who use sulfonylureas or insulin. Diabetes Care 29(2), 254-258 (2006).

7. Buzzai M, Jones RG, Amaravadi RK et al. Systemic treatment with the antidiabetic drug metformin selectively impairs p53-deficient tumor cell growth. Cancer Res. 67(14), 6745-6752 (2007).

8. Liu B, Fan Z, Edgerton SM, Yang X, Lind SE, Thor AD. Potent anti-proliferative effects of metformin on trastuzumab-resistant breast cancer cells via inhibition of erbB2/IGF-1 receptor interactions. Cell Cycle 10(17), 2959-2966 (2011).

9. Bodmer M, Becker C, Meier C, Jick SS, Meier CR. Use of metformin and the risk of ovarian cancer: a case-control analysis. Gynecol. Oncol. 123(2), 200-204 (2011).
10. Choi HJ, Kim TY, Chung N et al. The influence of the BRAF V600E mutation in thyroid cancer cell lines on the anticancer effects of 5-aminoimidazole-4-carboxamideribonucleoside (AICAR). J. Endocrinol. 211(1), 79-85 (2011).

11. Lin JN, Lin VC, Rau KM et al. Resveratrol modulates tumor cell proliferation and protein translation via SIRT1-dependent AMPK activation. J. Agric. Food Chem. 58(3), 15841592 (2010).

12. Wei S, Kulp SK, Chen CS. Energy restriction as an antitumor target of thiazolidinediones. J. Biol. Chem. 285(13), 9780-9791 (2010).

13. Chen $\mathrm{CH}$, Huang PH, Chu PC et al. Energy restriction-mimetic agents induce apoptosis in prostate cancer cells in part through epigenetic activation of KLF6 tumor suppressor gene expression. J. Biol. Chem. 286(12), 9968-9976 (2011).

14. Lin HY, Kuo YC, Weng YI et al. Activation of silenced tumor suppressor genes in prostate 
cancer cells by a novel energy restrictionmimetic agent. Prostate doi:10.1002/ pros.22530 (2012) (Epub ahead of print).

15. Rosano C. Molecular model of hexokinase binding to the outer mitochondrial membrane porin (VDAC1): implication for the design of new cancer therapies. Mitochondrion 11(3), 513-519 (2011).

16. Mathupala SP, Ko YH, Pedersen PL. Hexokinase II: cancer's double-edged sword acting as both facilitator and gatekeeper of malignancy when bound to mitochondria. Oncogene 25(34), 4777-4786 (2006).

17. Lee GH, Chae HJ, Kim HR. Monoamine carboxylate transporters are involved in BI-1associated cancer metastasis in HT1080 colon fibrosarcoma cells. Int. J. Oncol. 39(1), 209-216 (2011).

18. Milane L, Duan Z, Amiji M. Development of EGFR-targeted polymer blend nanocarriers for combination paclitaxel/lonidamine delivery to treat multi-drug resistance in human breast and ovarian tumor cells. Mol. Pharm. 8(1), 185-203 (2011).

19. De Lena M, Lorusso V, Latorre A et al. Paclitaxel, cisplatin and lonidamine in advanced ovarian cancer. A Phase II study. Eur. J. Cancer 37(3), 364-368 (2001).

20. Kim W, Yoon JH, Jeong JM et al. Apoptosisinducing antitumor efficacy of hexokinase II inhibitor in hepatocellular carcinoma. Mol. Cancer Ther. 6(9), 2554-2562 (2007).

21. Sanchez-Arago M, Cuezva JM. The bioenergetic signature of isogenic colon cancer cells predicts the cell death response to treatment with 3-bromopyruvate, iodoacetate or 5-fluorouracil. J. Transl. Med. 9, 19 (2011).

22. Buijs M, Vossen JA, Geschwind JF et al. Specificity of the anti-glycolytic activity of 3-bromopyruvate confirmed by FDG uptake in a rat model of breast cancer. Invest. New Drugs 27(2), 120-123 (2009).
23. Vander Heiden MG, Locasale JW, Swanson KD et al. Evidence for an alternative glycolytic pathway in rapidly proliferating cells. Science 329(5998), 1492-1499 (2010).

24. Cairns RA, Harris IS, Mak TW. Regulation of cancer cell metabolism. Nat. Rev. Cancer 11(2), 85-95 (2011).

25. Tennant DA, Duran RV, Gottlieb E. Targeting metabolic transformation for cancer therapy. Nat. Rev. Cancer 10 (4), 267-277 (2010).

26. Shimizu T, Okamoto I, Tamura K et al. Phase I clinical and pharmacokinetic study of the glucose-conjugated cytotoxic agent D-19575 (glufosfamide) in patients with solid tumors. Cancer Chemother. Pharmacol. 65(2), 243-250 (2010). 